\title{
Fatigue of Closed Cell Foams in Compression
}

\author{
Dan Zenkert ${ }^{1}$, and Magnus Burman \\ Department of Aeronautical and Vehicle Engineering \\ Kungliga Tekniska Högskolan (KTH) \\ SE - 10044 Stockholm, Sweden
}

Keywords: Cellular materials, foam, fatigue, compression

\begin{abstract}
This paper deals with fatigue of closed cell foams under compression loading. Testing is performed on three densities of Divinycell H-grade and three densities of Rohacell WF-grade foam under cyclic compression loading. The fatigue failure is in all cases described as localised crushing of cell layers. The two main observations from this are that the slopes of the stress-life curves are almost all different, both between the foam types and relative density. Secondly, the stress-life relation slopes are considerably flatter than corresponding data in tension. For one particular foam grade, the stress-life relation is almost flat.
\end{abstract}

\section{Introduction}

Many applications of sandwich structures in e.g. aerospace, automotive, marine and transportation, uses rigid cellular foams as load carrying core materials. Therefore, the mechanical properties of this class of materials are essential for the design of such structures. Static properties of foams are currently well documented, whereas fracture and fatigue properties still require more testing, models and validation. Cellular materials are also quite different from their solid counterparts due to their inherent micro-structure, being a 3dimensional network of thin membranes and rods building up a volume of stochastically formed closed cells. Thus, depending on the scale, a foam can be regarded as a solid continuum or a heterogeneous structure.

The present study builds on the work performed in [1] and [2], where only tension fatigue was studied. In [1], tension fatigue experiments were performed on the same materials as used herein, at a load ration $R=0.1$. By using previously measured crack propagation rate data, a simple life prediction model was proposed based on an initial flaw approach. This model predicts the unnotched fatigue behaviour in tension for the investigated foams. However, the model was not really complete, but requires one test to find a reference point for the stress-life curve. The tensile stress-strain relations were normalised with the density and shown to form a generic relation. The density normalisation was performed using

$$
\bar{x}=\alpha \bar{\rho}^{n}
$$

where $\bar{x}$ is some mechanical property of the foam normalised with its value for the material bulk property of which the cell edges and faces are made of, and $\bar{\rho}$ is the foam density normalised with the bulk density of the material. It was shown that other properties, like fracture toughness, also scale similarly well using this approach. It was then found that the tensile fatigue data could be normalised this way, enabling the construction of generic,

\footnotetext{
${ }^{1}$ Corresponding author, danz@kth.se
} 
material specific, but density independent, stress-life relations. In a follow-up study [2], the fatigue behaviour in tension, compression and shear of one class of structural foams (Rohacell) was performed. One conclusion from this was that the stress-life relations were different for the different load cases, generally with lower slope for compression and shear, than in tension. However, for the highest density used in that study (WF200), the slopes were all the same for all load cases, indicating that for this relatively high density foam, the failure mechanism must be the same independent of loading condition. For lower densities, the failure mechanism in compression and shear must then be different than in tension, possibly being connected to localised cell wall buckling.

The aim of this paper is to compare compression fatigue data for two different structural foams. For each material type three different densities were investigated. The compression data presented here for the Rohacell WF foams are directly reproduced from [2] but are included for clarity but moreover in order to make proper comparisons. There is not much reported in the open literature on compression fatigue of foams. McCullough et al [3] tested aluminium foams in both tension-tension and compression-compression fatigue. They scale properties of these foams with a power law relation of the type given in eq.(1) with an exponent equal to 1.5. Although the results are not given in terms of a Basquins' law it is found that the slope of the S-N curve is considerably lower in the compression-compression fatigue case. It was also found that high density and low density foams appear to have very similar S-N curves, though with different amplitude. They also argue that the fatigue degradation mechanism is not due to cracking events in the foam but rather progressive accumulation of strain by material ratchetting analogous to creep. Once the accumulated strain equals the monotonic yield strain the rate of accumulation increases at least one order of magnitude. This leads to the formation of a complete crush band in compression or a rupture band in tension. This is supported also by the fact that the fatigue life is found to be notch insensitive for the metal foams used in their work.

\section{Materials}

In this investigation two closed cell foams have been used; Divinycell H-grade and Rohacell WF-grade. Three densities of each material has been used given by the notation of each foam, e.g. H60 has a density of $60 \mathrm{~kg} / \mathrm{m}^{3}$. Detailed information and their static properties according to the manufacturers can be found in refs. [4,5]. Both foams are used in various structural applications as load carrying core materials in sandwich panels. These two foams are slightly different in mechanical performance. The Rohacell foam has slightly higher static mechanical properties per unit weight but has considerably lower strain to failure and is thus to be considered more brittle. Although both foams are closed cell structures, a portion of the solid material is concentrated around the edges (junction between the walls) and they thus do not behave like a perfect closed cell foam implying that the exponent in eq.(1) should be equal to 1 if one follows the theory according to Gibson and Ashby [6]. By fitting handbook data of static properties, one finds the exponent in eq.(1) to be equal to 1.1 to 1.2 [1].

\section{Static properties}

The static tests of foams were performed using the same test up as in the compressioncompression fatigue testing, which is described below. Fig.1 shows compressive stress-strain 
curves for all materials. These were all measured using the same test set-up as used in the fatigue testing.

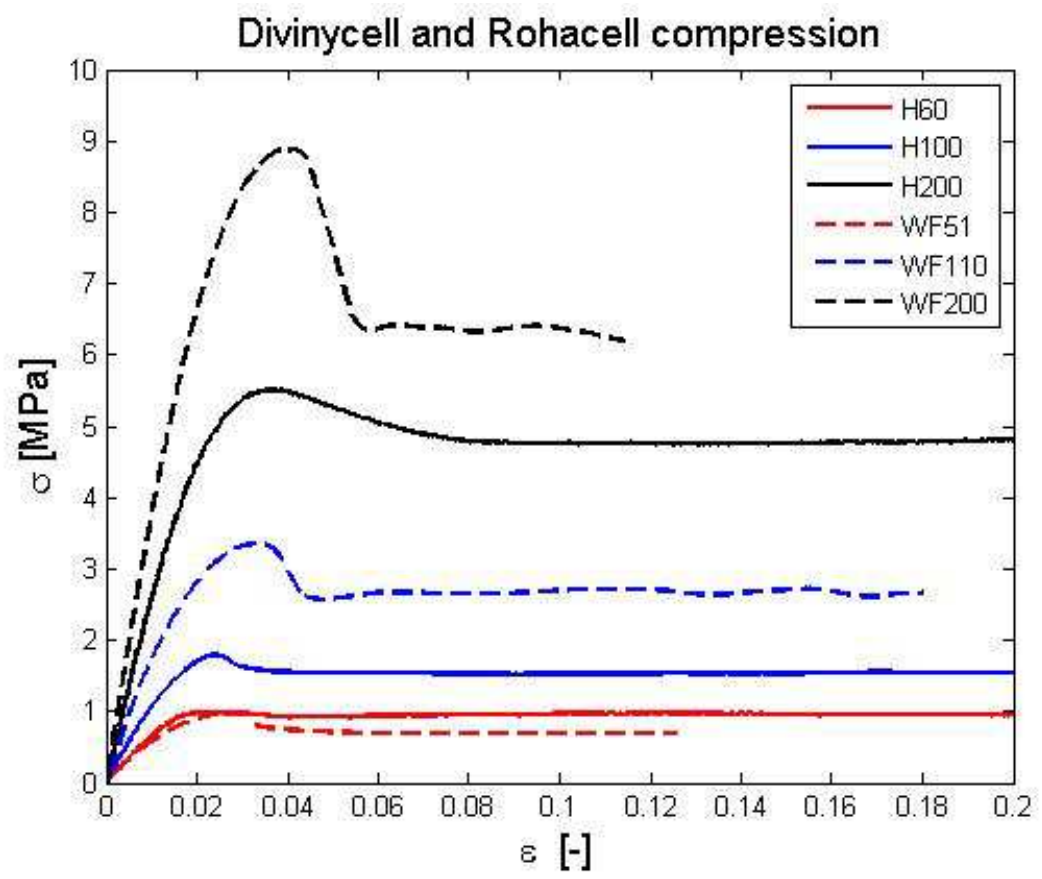

Figure 1 Typical stress-strain relations for $\mathrm{H60}, \mathrm{H} 100$ and $\mathrm{H} 200$ (solid lines) and WF51, WF110 and WF200 (dashed lines).

As seen from Fig.1, the compressive stress-strain relation can be defined by a linear part, followed by a non-linear part, a distinct peak followed by a small stress drop and then a so called plateau level. The manufacturers almost always identify this peak stress in the stressstrain relation as the compressive strength. Compression failure initiates at peak stress by the formation of crush bands. This implies that one (weak) layer of cells buckles and/or fails, collapses and thus densifies. The first crush band then has a thickness of approximately one cell size. Then, an adjacent cell layer collapses, widening the crush band. Thus, the plateau stress level is described by progressive crush band formation. Eventually, the crush band covers the entire specimen and the material reaches the so called densification point, where the stiffness starts of increase again. This part has been truncated from Fig.1 and occurs at relatively high strains, in the order of 0.5-0.8 depending on the density of the foam.

We have chosen the yield points in compression as the $1 \%$ offset stress. There is no particular rationale behind this choice except that these points provide good cut-off stress limits for the fatigue results described in the following sections. The measured material data for the considered materials are given in Table 1. 


\begin{tabular}{|c|c|c|c|c|c|c|}
\hline & H60 & H100 & H200 & WF51 & WF110 & WF200 \\
\hline E [MPa] & 78 & 126 & 304 & 75 & 200 & 395 \\
\hline$\sigma_{1 \%, \text { comp }}[\mathrm{MPa}]$ & 0.9 & 1.6 & 5.1 & 0.85 & 3.3 & 7.8 \\
\hline$\sigma_{\text {ult, comp }}[\mathrm{MPa}]$ & 1.0 & 1.8 & 5.6 & 1.0 & 3.5 & 8.8 \\
\hline$\sigma_{u l t, \text { tension }}[\mathrm{MPa}] 1$ & 1.8 & 3.3 & 7.1 & 1.6 & 3.4 & 7.4 \\
\hline$\beta[-]$ & 14 & 27 & 63 & 24 & 24 & 15 \\
\hline
\end{tabular}

Table 1 Measured material data for Divinycell $\mathrm{H}$ and Rohacell WF materials. Material data in tension are taken from [1]. $\beta$ is defined in results section.

In [1] it was found that the tensile stress-strain relations could be normalised with the relative density, as given in eq.(1). Any attempt to do so with the compressive data is impossible. The reason for this is not absolutely clear, but can possibly be explained by the following; the cell wall material is the same for all densities. Higher densities have smaller cells and thicker cell edges and cell walls. Following the procedures by Gibson and Ashby [6], it can be identified that for an idealised open cell foam the cell edge buckling stress is proportional to the relative density square $\left(\bar{\rho}^{2}\right)$. A similar reasoning for an idealised closed cell foam would give that cell wall buckling is proportional to the relative density cube $\left(\bar{\rho}^{3}\right)$. Thus, cell wall buckling is most likely the dominating triggering mechanism for failure in compression for the low density foam, but not for higher densities.

\section{Compression-compression fatigue testing}

The compression-compression fatigue tests were performed using an axi-symmetric dog bone specimen, described in ASTM D1623-78 "Tensile and tensile adhesion properties of rigid cellular plastics" [7]. This set-up is similar to that used for the tensile fatigue testing reported in [1]. The specimens were cut from blocks of foam core, bonded between two aluminium cylinders and a waist was milled to the correct shape and size in a lathe. Thus, this testing is done in the thickness direction of the foam material block. The test rig with a specimen is shown in Fig.2.

Static tests were performed in compression under a constant deformation rate of $2 \mathrm{~mm} / \mathrm{min}$ at room temperature using the same specimen type. The fatigue tests were performed under a load controlled sinusoidal cycle using a servo hydraulic testing machine. The load ratio used was in all cases $R=10, R=\sigma_{\min } / \sigma_{\max }$ and a testing frequency of $5 \mathrm{~Hz}$. $\sigma_{\min }$ is the peak negative stress in the load cycle and thus $\sigma_{\max }=0.1 \sigma_{\min }$, which still remains negative. The highest compressive stress $\left(\sigma_{\min }\right)$ used in the fatigue testing is set to be approximately equal to the yield stress in compression according to Table 1.

The fatigue life of the specimens is characterised as described in the following. 

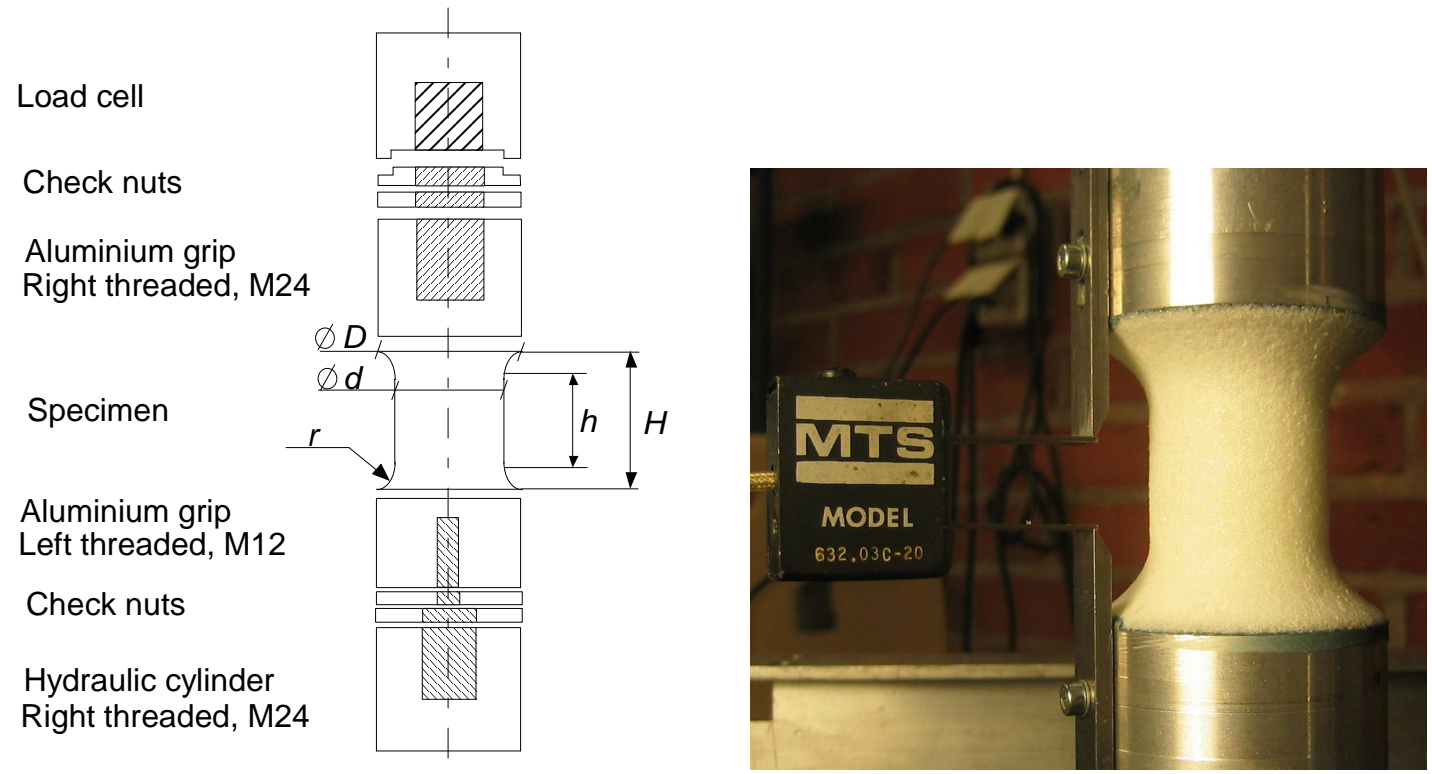

Figure 2 (a) Schematic drawing of the test set-up tensile fatigue specimen. Dimension used $D=50 \mathrm{~mm}, d=30 \mathrm{~mm}, H=50 \mathrm{~mm}$, and $h=32 \mathrm{~mm}$. (b) Photograph of WF110 specimen mounted in the testing machine.

A clip-gauge was mounted to the specimen measuring the displacement in each load cycle. The test was interrupted when a permanent strain of $2 \%$ was reached $(1 \mathrm{~mm}$ permanent deformation). At such permanent strain it was found that a crush band had formed in the specimen. The thickness of this crush band was at least one layer of cells, and this was taken as the failure criterion. This is similar to the approach used by McCullough et al [3]. Every specimen was inspected visually to ensure that a crush band had formed once the test was interrupted. This could also be seen when plotting the maximum or minimum displacement of the clip-gauge during the test. In Figs.3a-f the clip-gauge displacements are shown for selected test specimens, one for each material and density. The sudden drop in displacement as seen herein are all associated with the formation of a crush band in the specimens. We have intentionally chosen specimens with different fatigue life in order to illustrate that this failure mode is independent of fatigue loading amplitude and thus fatigue life. For the H-grade materials (Figs.3a-c) there seems to be a little bit more creep deformation prior to the crush band collapse in comparison to the WF-materials (Figs.3d-f).

There is one interesting result shown for a WF110 specimen in Fig.3e. Here, there are two displacements drops, the first one occurring at approximately $3 \cdot 10^{5}$ load cycles. The drop is approximately $0.4 \mathrm{~mm}$, which corresponds quite well to the average cell size of the material. The displacement drop thus indicates that one layer of cells in the specimens has crushed and compacted. There is a clear indication of a second displacement drop some 20000 cycles later, which most probably indicates that an adjacent layer of cells is crushing. 


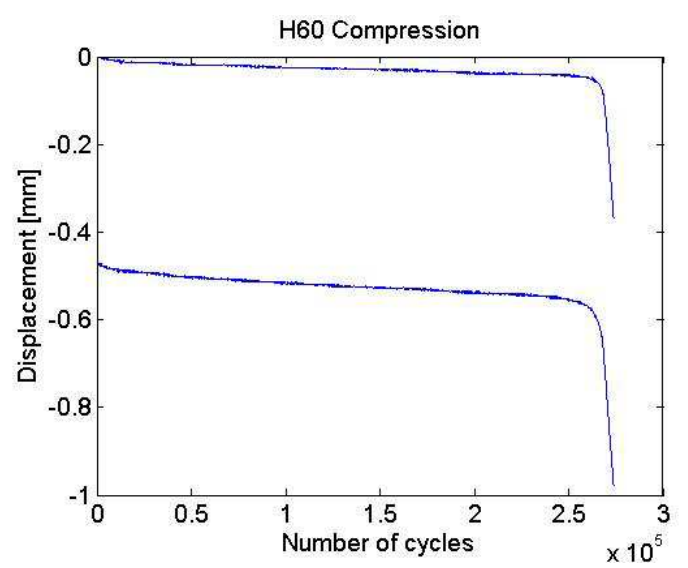

(a)

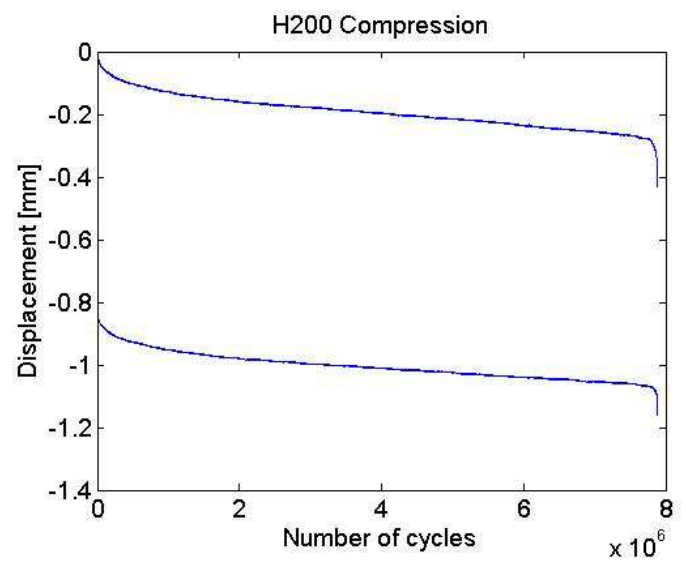

(c)

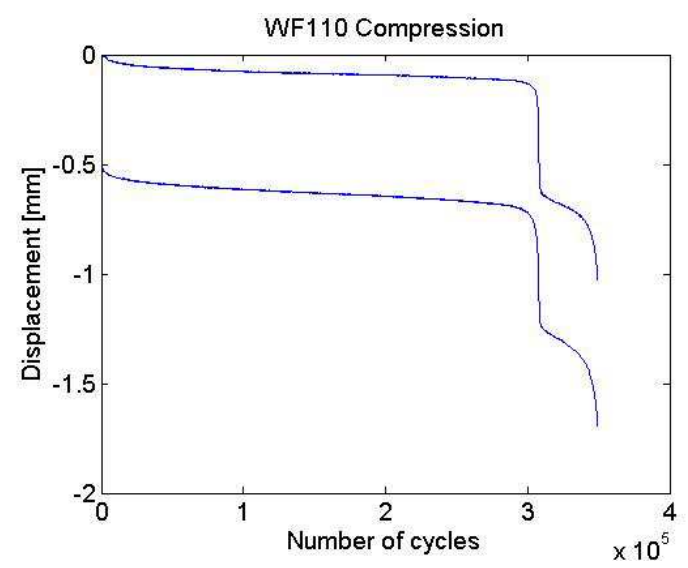

(e)

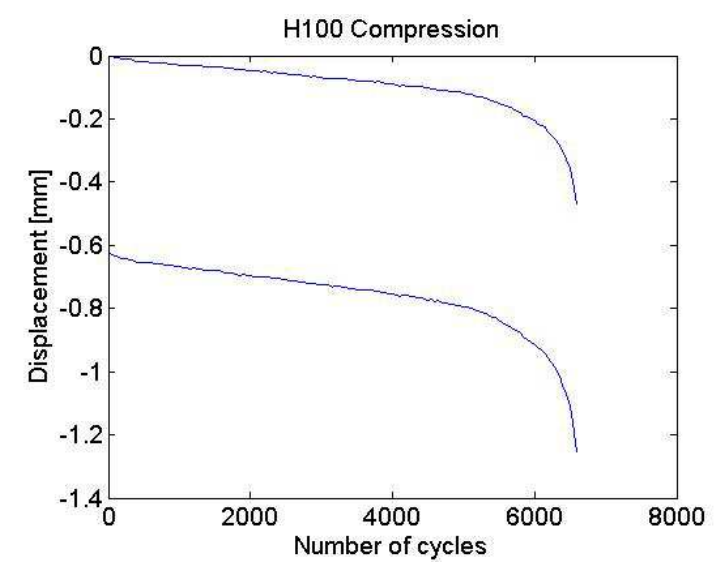

(b)

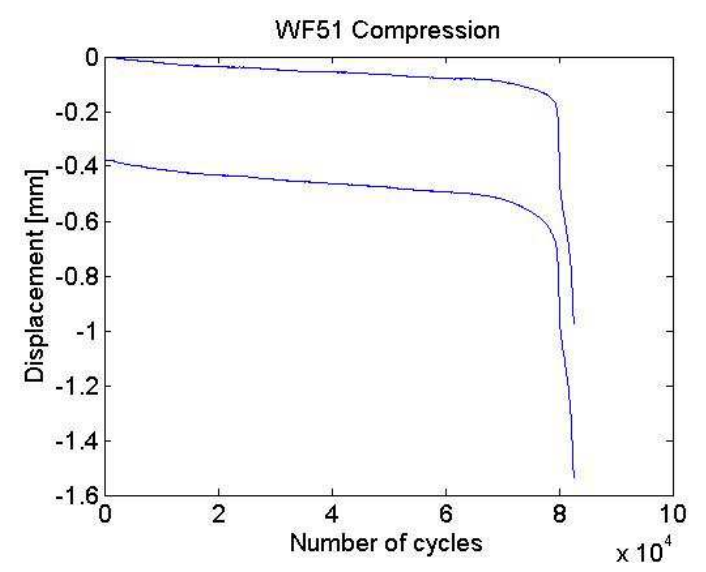

(d)

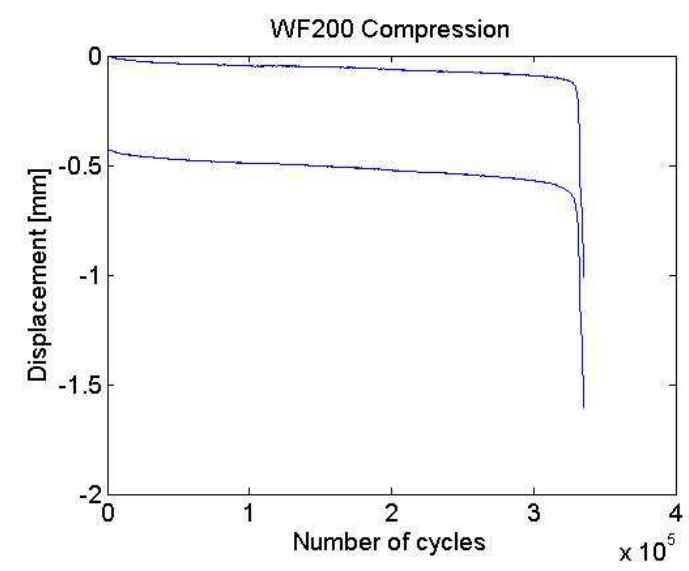

(f)

Figure 3 Maximum and minimum displacement versus number of cycles in a compressioncompression test. Specimen of (a) H60, (b) H100, (c) H200, (d) WF51, (e) WF110 and (f) WF200 material

The failure is indicated by the formation of a crush band leading to a sudden displacement change in the test. Photographs of some failed test specimens are shown in Figs.4 and 5. Fig.4 shows photographs taken from the surfaces of fractured specimens. Here the crush bands are clearly indicated and can easily be observed visually. They are furthermore clearly confined to one or a few layers of cells making the crush band thin. This was the case for all WFmaterials. For the H-materials the failures were a little more difficult see on the surface. The 
crush bands were generally thicker and did not seem to be confined to one layer of cells, but to a number of cells.

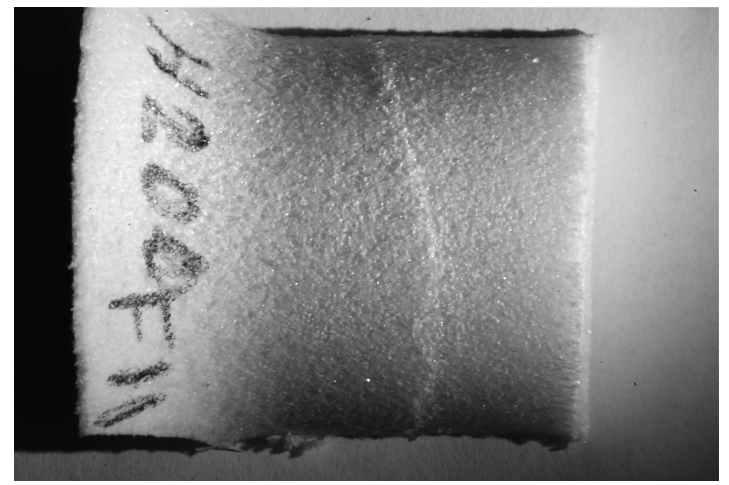

(a)

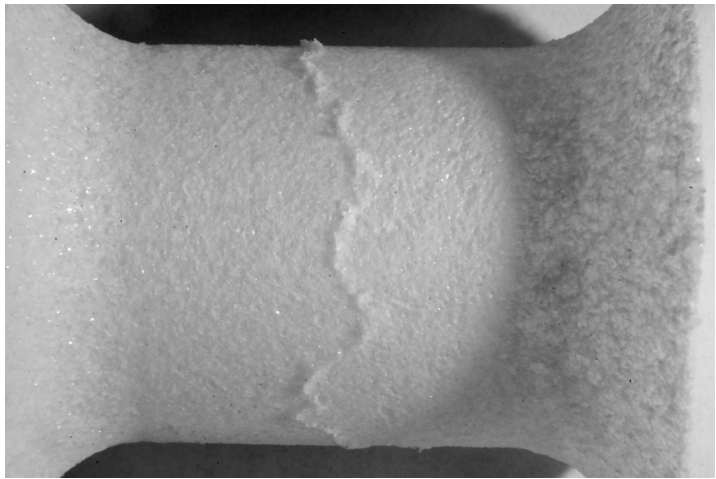

(b)

Figure 4 Failure in compression fatigue of (a) H200 and (b) WF110 as seen from the surface of the specimens.

Slices of 2-4 mm thickness were cut from the specimens and photographed with back light, shown in Fig.5. Though in some cases hard to see, they all show regions of undamaged cells appearing light due to light transmitting thought the thin walls. In the damaged areas, the cells are crushed and compacted, indicated by darker areas in the photographs. The exception is the H200 material, in Fig.5c, which had to be photographed in raking light as to visualize the crushed zone. Here it appears lighter due to more reflection.

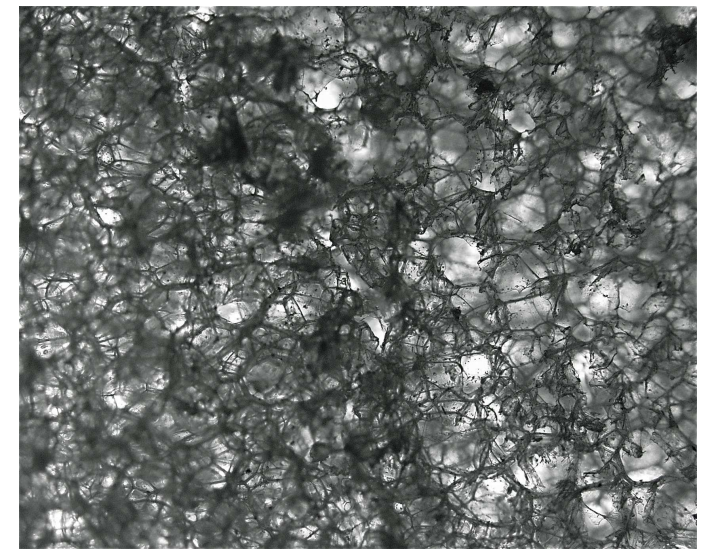

(a)

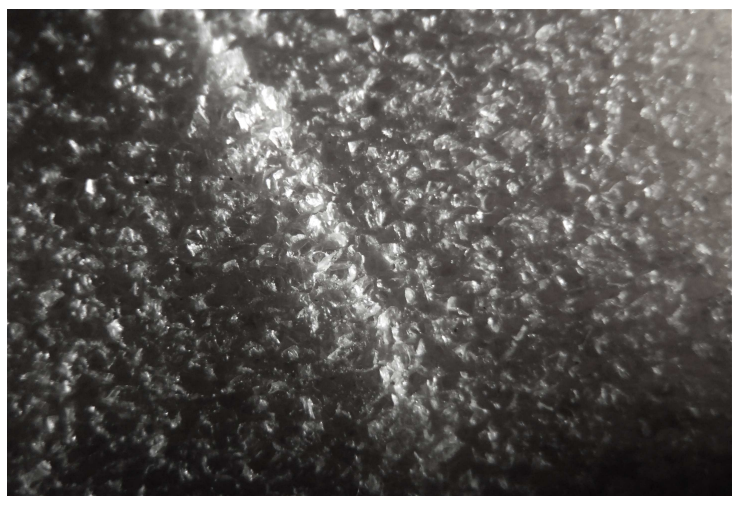

(c)

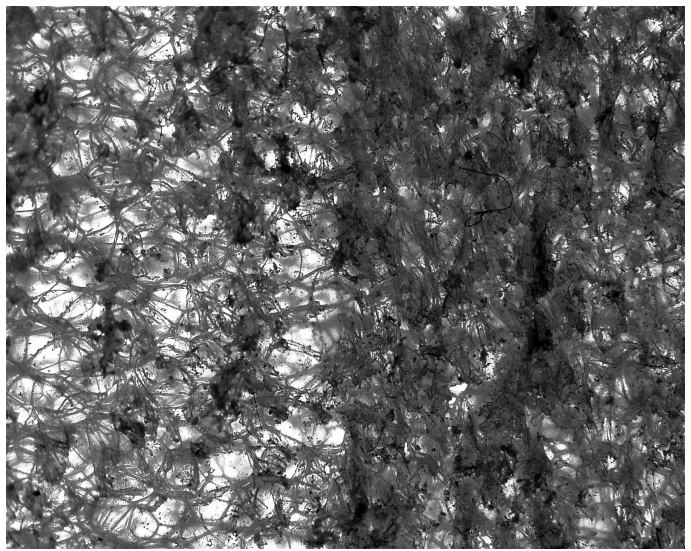

(b)

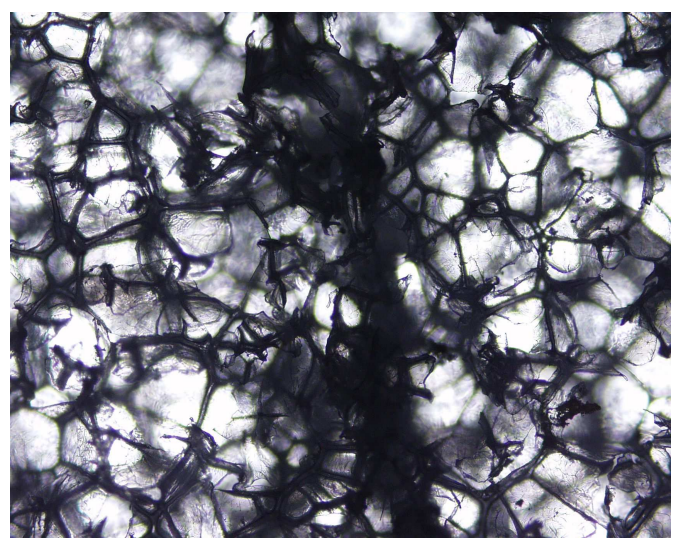

(d) 


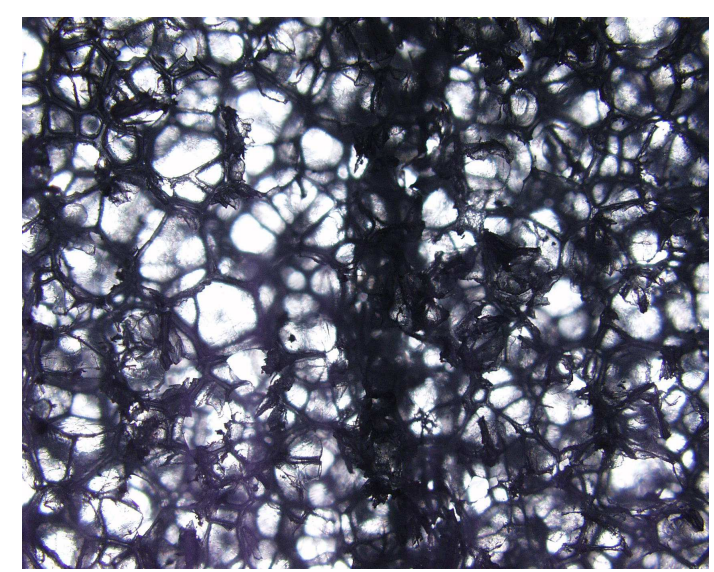

(e)



(f)

Figure 5 Failure in compression fatigue of (a) H60, (b) H100, (c) H200, (d) WF51, (e) WF110 and (f) WF200 material.

\section{Results and discussion}

The compression fatigue results are summarised in Figs.6 and 7. The dashed lines indicate the static yield stress of the materials, the markers indicate actual fatigue test results and the solids lines are curve fits to the experiments. The curve fits are based on the results from fatigue tests performed at a level below the static yield point, i.e. fatigue test data from specimens run at or above the yield point are omitted in the curve fit, but they are still included in the graphs as test points. The reason is that at yield stress (or above) the scatter in test results is very large (see e.g. H100). For the H200 the results from the fatigue tests performed at the yield stress had to be included in the curve fit when slope of the stress life relation for this material is extremely flat.

In [1] and [2] it was rationalised that the stress-life relation basically is bi-linear, with a cutoff at the yield stress. The regime above this level is associated with low-cycle fatigue and below is the high-cycle regime. For the H-materials, this transition from the low-cycle to the high-cycle regime appears at approximately $10^{4}$ load cycles and for the WF-materials at approximately $10^{2}$ load cycles.

The stress-life data for the high-cycle regime is herein fitted to a Basquin's law type relation that reads

$$
\Delta \sigma=B(N)^{-1 / \beta}
$$

where $\Delta \sigma$ is the stress range, $N$ the number of cycles to failure, $B$ a fitting constant and $-1 / \beta$ the slope of the relation. The stress value plotted in Figs. 6 is the maximum compressive stress given by $\sigma_{\max }=\Delta \sigma /(1-R)$. When the slope of the stress-life relation is discussed, the value referred to is $\beta$. A high value of $\beta$ means a low slope and a low $\beta$ means high slope. The values of $\beta$ are given in Table 1 for all materials but are further discussed in more detail in the following. 
For the H60 and H100-materials (Fig.6) the fatigue results show fairly low scatter, except above yield stress. The slope of the stress-life relations are 14 and 27, respectively, as also given in Table 1. The H200 material exhibits a more unexpected behaviour. The slope of the stress-life curve is very low with a best fit value of $\beta$ equal to 63. Basically it means that a very small change in the load amplitude changes the fatigue life from only a few hundred to several millions of cycles to failure. For the $\mathrm{H}$-materials, the fatigue life curves in tension all had the same slope, approximately $\beta=6$ [1]. In compression, all the H-grade densities exhibit very different behaviour with much lower slopes of the fatigue life curve and apparently with decreasing slope for increasing density. For H200 the situation is clearly quite extreme. The compressive and tensile static strengths are almost equal, but the slopes in the fatigue relations are very different.

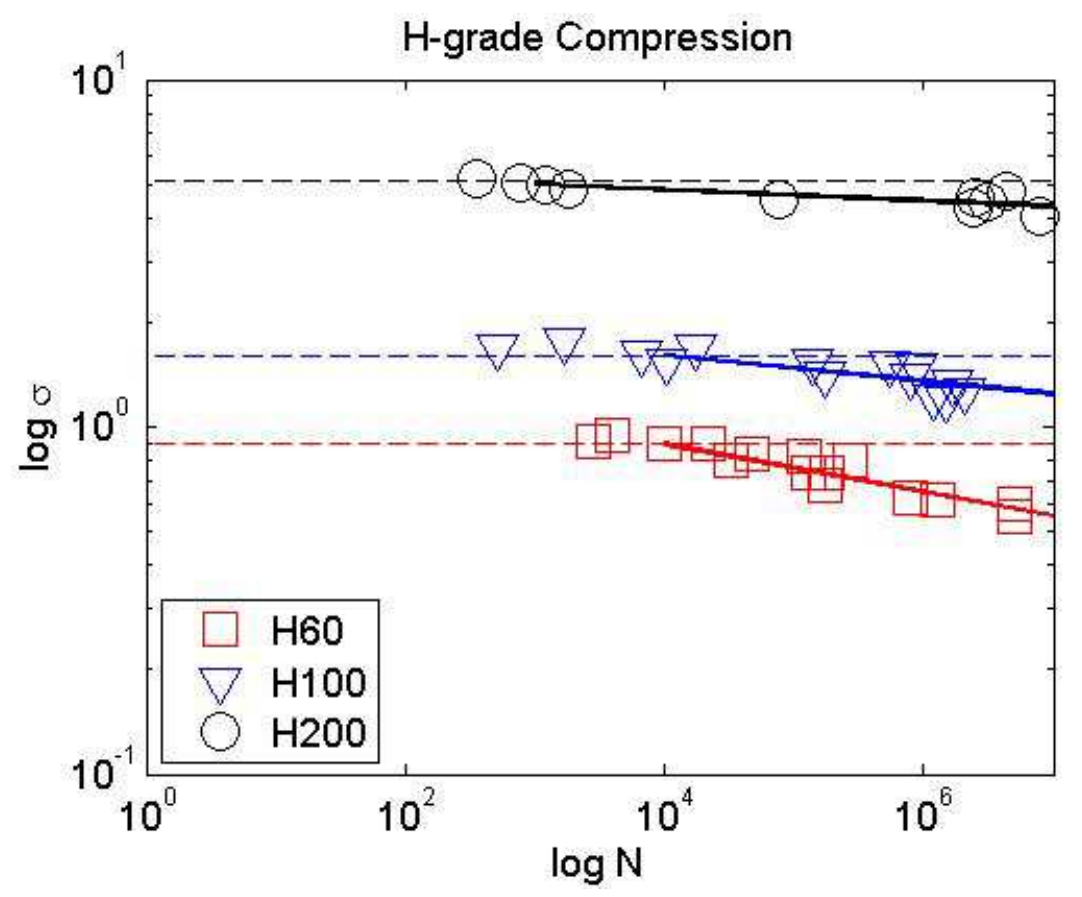

Figure 6 Fatigue results in compression for $\mathrm{H} 60, \mathrm{H} 100$ and $\mathrm{H} 200$

The slopes of the stress-life relation are quite different for the materials studied herein. In compression fatigue, the H-material exhibits lower fatigue life slopes with increasing density, 14, 27 and 63 for H60, H100 and H200 respectively. In comparison one may observe that the slopes of the tensile stress-life relations were approximately 6 [1] and independent of material density. There is no obvious explanation for this difference in stress-life response due loading.

For the H60 and H100 materials, the strength is considerably higher in tension than in compression and this relation is also seen in the fatigue performance; for the same stress level the fatigue life is higher for tensile loads than for compressive. For H200, the strengths are approximately equal in tension and compression, but here a better fatigue performance in compression is observed due to the lower slope of the stress-life curve.

It is not clear why the slopes of the fatigue relations are density dependent. As stated above the compressive strength is lower than the tensile strength for the lower density foams. A feasible explanation for this is that in tension, cell walls and edges rupture. In compression, cell walls buckle and thus initiate the formation of crush bands. It is likely that the same 
mechanisms are active also in fatigue. For higher density foams the compressive strength is equal to, or higher, than the tensile strength. Higher density foams have smaller cells and thicker cell edges and walls. Following the procedures by Gibson and Ashby [6], it can be identified that for an idealised closed cell the cell wall buckling load is proportional to the relative density cube $\left(\bar{\rho}^{3}\right)$. Thus, buckling is most likely the dominating triggering mechanism for failure in compression for the low density foam, but not for higher densities.

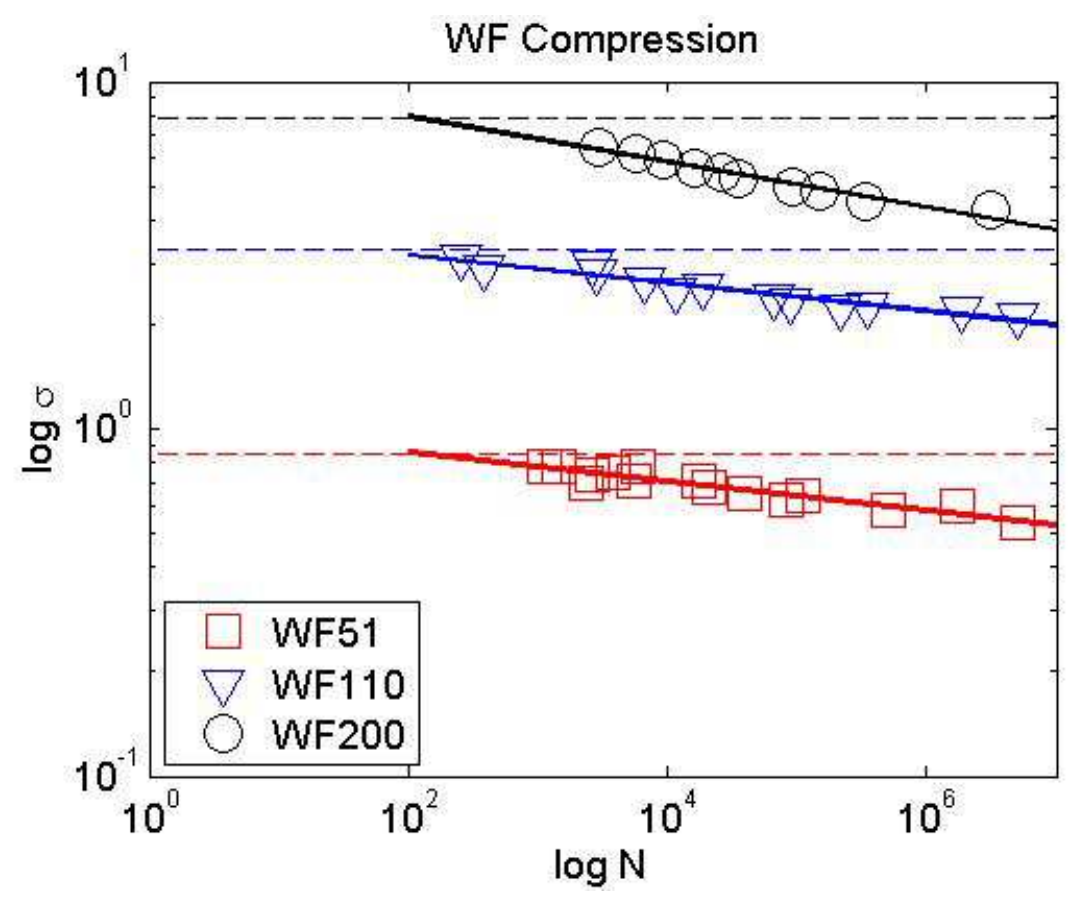

Figure 7 Fatigue results in compression for for WF51, WF110 and WF200

The fatigue results for all WF-materials in Fig.7 show really low scatter. Best fits of the data to eq.(2) gives slopes of 24, 24 and 15 for WF51, WF110 and WF200, respectively (see also Table 1). In tension, the fatigue life curves all had the same slope, with approximately $\beta=13$ [1]. For WF200 the stress-life relations in tension and compression are thus very similar, almost the same slope but with slightly higher amplitude in compression. This relation gives some indication that the failure process and failure mode on a microscopic level could be the same for the two different loading conditions. The fatigue life relation in shear for WF200 also exhibits the same slope [2], whereas for the lower densities slopes differ between the different loading conditions [2].

The compression results of H100 foam Fig.6 is re-plotted in Fig. 8 together with tensile fatigue data from [1]. As noted in the graph and previously discussed the H100 material has higher strength in tension than in compression. On the other hand, in the high-cycle fatigue regime, there appears to be a point around $10^{5}$ load cycles where the material has approximately equal life for equal stress in tension and compression. This has potentially some implications. If this foam is subjected to fatigue loading under $R=-1$, one would expect compressive failure at high load amplitudes (small number of cycles to failure), but potentially tensile failure for lower loads (when the total number of cycles to failure is expected to the above $10^{5}$ ). For the other densities and also for the WF material, the situation is similar as in Fig.8, although the individual slopes and amplitudes differ. For H60, the fatigue properties are always higher in tension than in compression and the cross-over that is seen in Fig.8 will occur at cycles above 
$10^{7}$ cycles. For $\mathrm{H} 200$, the fatigue properties (in terms of stress versus number of cycles) are always better in compression than in tension, since the static strength is higher in compression and the slope is lower. For WF51 the behaviour is similar to H60 but for WF110 and WF200 it is similar to $\mathrm{H} 200$ always being better in compression.

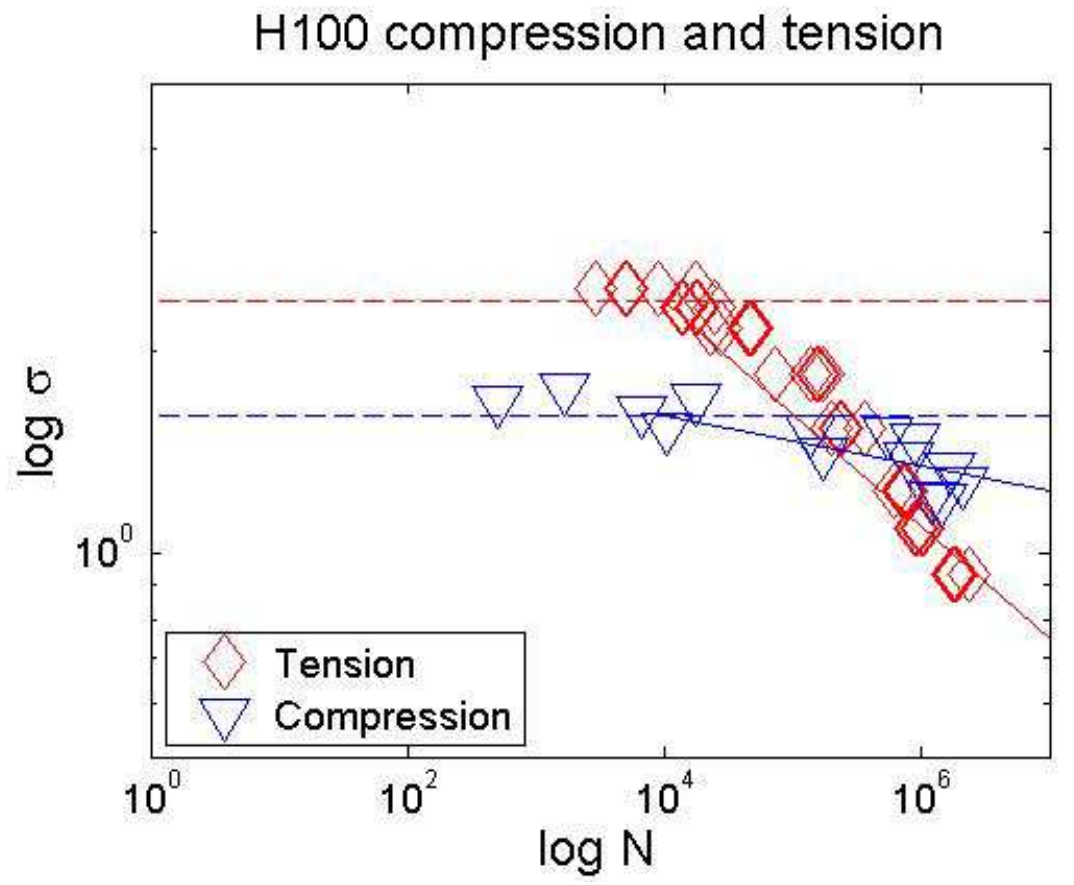

Figure 8 Fatigue results in compression and tension $\mathrm{H} 100$

\section{Conclusions}

Fatigue testing in compression was performed for two cellular foam cores used in structural applications. For each material, three different densities were tested. It was found that all material exhibit in principal the same failure mode in fatigue corresponding to the formation of a crush band.

Stress-life relations could be obtained from the test data with relatively low scatter overall. The data was fitted to a Basquin's law type relation from which the slope of the stress-life relation was obtained. It was found that this slope differs between the materials but also between densities within one class of material.

By comparing the compression data with previously measured fatigue data in tension one finds that the slope of the stress-life relation in compression is considerably lower than in tension, implying that the fatigue behaviour is better in compression than in tension.

\section{Acknowledgements}

The financial support for this investigation has been provided by The Office of Naval Research (ONR) through programme officer Dr. Yapa D.S. Rajapakse (Grant No. N0001407-1-0344). Evonik GmbH and DIAB AB are acknowledged for supplying the materials. Special thanks to Anders Bäckman and Bo Magnusson for help with the manufacturing of the test specimens and the fatigue testing. 


\section{References}

[1] Zenkert D., Shipsha A. and Burman M., "Fatigue of Closed Cell Foams", Journal of Sandwich Structures and Materials, Vol 8 (6), 2006, pp 517-538.

[2] Zenkert D. and Burman M., "Tension, Compression and Shear Fatigue of a Closed Foam”, Composite Science and Technology, Vol 69, 2009, pp 785-792

[3] McCullough K.Y.G., Fleck N.A. and Ashby M.F., "The stress-life fatigue behaviour of aluminium alloy foams", Fatigue and Fracture of Engineering Materials and Structures, Vol.23(3), 2000, pp 199-208

[4] Divinycell H-grade, Technical Manual, DIAB AB, Laholm, Sweden, www.diabgroup.com.

[5] Rohacell WF, Evonik Industries, www.rohacell.com

[6] Gibson L.J. and Ashby M.F., Cellular Solids - Structure and Properties, 2:nd edition, Cambridge University Press, Cambridge, UK, 1997.

[7] Annual Book of the ASTM Standards, American Society for Testing and Materials, Philadelphia, PA. 